\title{
AERODYNAMIC PERFORMANCE OF THE DeSiReH HIGH-LIFT LAMINAR WING AT FREE FLIGHT AND ETW IN-TUNNEL CONDITIONS
}

\section{S. Bosnyakov, E. Kazhan, I. Kursakov, S. Matyash, S. Mikhaylov, and A. Lysenkov}

Central Aerohydrodynamic Institute (TsAGI)

1 Zhukovsky Str., Zhukovsky, Moscow Region 140180, Russia

\begin{abstract}
The current research concerns a half-model high-lift configuration inside the European Transonic Wind Tunnel (ETW) at landing regime. The influence of the wind-tunnel walls (both slotted and closed) is investigated and the numerical results are compared with measured data. The investigated model is a three-element landing configuration with Krueger device and flap. All calculations are performed on structured grids using EWT-TsAGI code. The computed in-tunnel results are in good agreement with uncorrected experimental data, with maximum lift predicted at the same angle of attack. The slotted wall configuration produces less wall interference than the closed wall configuration.
\end{abstract}

\section{INTRODUCTION}

The numerical simulation of the flow over high-lift configurations is still a challenging task. From geometrical point of view, its complexity requires a correct description of a number of multiscale processes such as wakes, flow separation, laminar/turbulent transition, and shock/boundary-layer interaction. Furthermore, at higher incidences angles the flow becomes essentially nonstationary.

For the last decades, significant progress was achieved in the calculations of such a task. In the EC projects EUROLIFT [1,2], EUROLIFT II [3, 4], DeSiReH, the complexity of the poblems solved grows. For example, one of the goals of EUROLIFT II project was the investigation of installation effects inside the cryogenic ETW, in which the experiments at high Reynolds numbers are conducted [4].

The work presented is performed in the frame of DeSiReH project (7th Framework Programme) funded by EC. This project intends the realization of the 
ACARE Vision 2020 for significantly greener aircraft and a reduced time to market by improving the aerodynamics of the high-lift system. This should be achieved by considering - at the same time and in coordinated approach - the numerical design methodology, the measurement techniques for cryogenic conditions for an advanced laminar wing design to be performed in DeSiReH. The final aerodynamic tests of the high-lift aircraft model were performed in ETW. The main goal of TsAGI team was to estimate the effects of wind-tunnel walls on the model characteristics. In order to achieve this goal, three series of computations were done. There are calculations for the model in a free flight environment and for the model installed in the wind tunnel both with open and closed slots.

\section{DESCRIPTION OF THE METHODOLOGY FOR THE COMPUTATIONAL TOOLS}

TsAGI is working with their structured multiblock in-house flow solver. The flow solver contains both explicit and implicit time stepping methods that can be combined with local time stepping for steady state problems. For high lift flows and moderate incidences, a time-averaged approach based on Reynoldsaveraged Navier-Stokes (RANS) equations is acceptable since the averaged flow field is in general steady. At higher incidences and beyond maximum lift, nonstationary processes with interactions between separated zones and vortex sheets past the wing become essential to be resolved time accurately with unsteady RANS (URANS). Explicit time stepping for time accurate problems are accurate but too expensive due to the small time steps enforced by the small grid cells in boundary layers and wakes. Implicit schemes, on the other hand, are good for multiscale problems but they often show poor quality for nonstationary flows.

A possible way to resolve this contradiction is to use a zonal method. In this approach, flow zones with very small scales of physical processes (mainly, near wall parts of boundary layers) are calculated using implicit time integration, while the other part of the flow is calculated using explicit integration. As a result, nonstationary processes in the inviscid core of the flow are simulated with a high quality. In the inner part of the boundary layers, an implicit scheme is used that propagates the information across the boundary layer and nonstationary processes are mainly described by the processes that take place in inviscid core of flow.

In the explicit part of the computational domain, local time steps are used. To maintain time accuracy, multiple local time steps are taken in the smaller cells. This idea was first published in [5] and is illustrated in Fig. 1.

The smallest time step $\left(\tau_{\min }=\min _{i} \tau_{i}^{\text {stab }}\right)$ is typically much smaller than the largest time step, $\tau_{\min } \ll \tau_{\max }=\max _{i} \tau_{i}$ on a nonuniform grid. In the current 


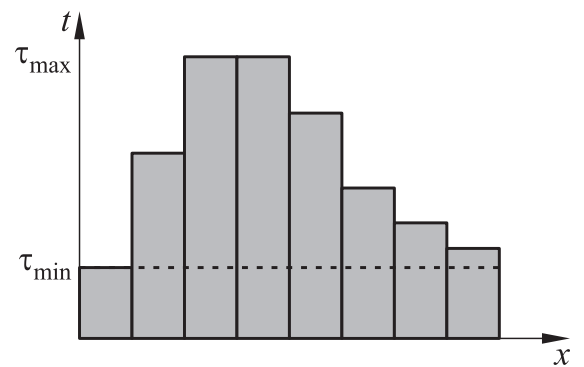

(a)

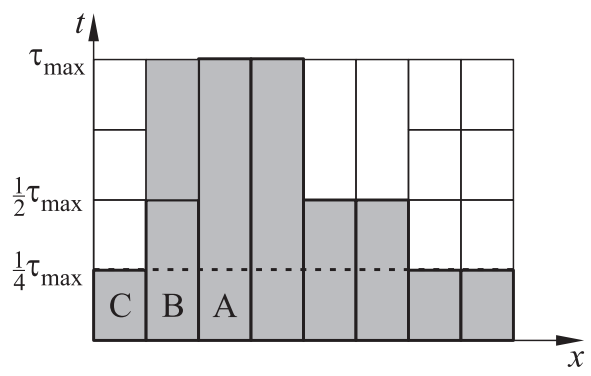

(b)

Figure 1 Global, local, and fractional time stepping

work, a method of fractional time stepping is proposed. The idea of fractional time stepping is that the calculation in each cell is performed with the local time step. But the numbers of local time steps are different in different cells and they are chosen so that all the cells reach the same physical time (Fig. 1b). When the same time has been reached, it corresponds to the completion of a global time step. For example, if a local time step in the cell $A$ is equal to $\tau_{\max }$, in $B$ to $\tau_{\max } / 2$, and in $C$ to $\tau_{\max } / 4$, then, during one global time step, one should perform one local time step in the cell $A$, two local time steps in the cell $B$, and four local time steps in the cell $C$. Therefore, the global time step in each cell is divided (fragmented) into smaller local time steps so that the local time steps satisfy the local restriction on time step in the given cell.

In the current research, the calculations for incidence angles below the stall were done with implicit scheme and local time spepping. At flow regimes beyond the maximum lift, the calculated strategy was more complicated: the field computed with implicit scheme and local time spepping was used as initial state for zonal method. Detailed discussion of the implementation of zonal approach in DeSiReH project can be found in [6].

\section{COMPUTATIONAL SETUP}

\subsection{Geometry Description}

The geometrical description is provided by the file partners of TsAGI in DeSiReH project. The configuration consists of a high-lift wing and a fuselage. The model is mounted on to a peniche and is presented in Fig. 2.

The wing of the model is supplied with deflected Krueger flap and deflected trailing edge flap (Fig. 3). The deflection angles of the leading and trailing edge devices correspond to a landing setting. 


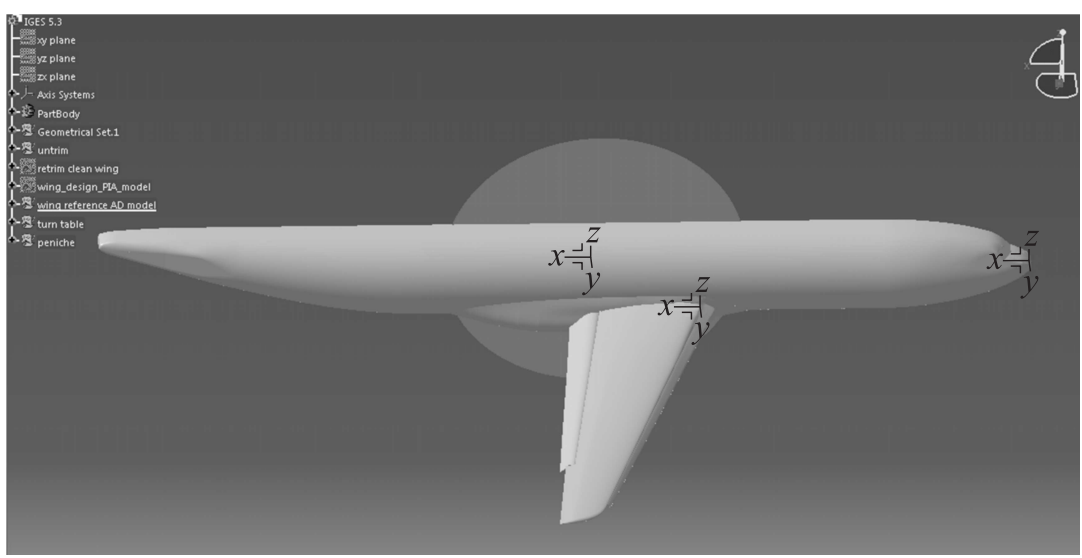

Figure 2 Reference configuration (wind tunnel model)

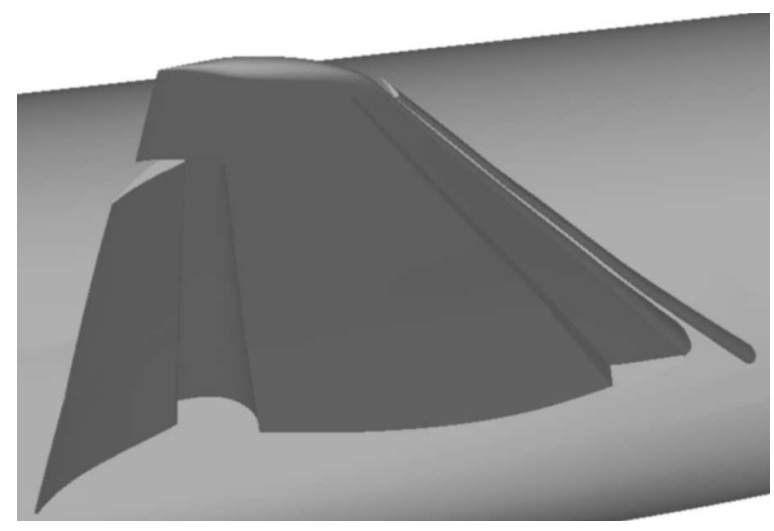

Figure 3 Wing with deflected flaps

The lower wing surface contains a cutout to store the moveable leading edge devices during the cruise flight. The geometrical description corresponds to the wind tunnel model which is to be tested in ETW, Cologne. The representation of the wind tunnel model is combined with a representation of the ETW. The combined representation is considered in two variants. The wind tunnel can operate with a test section that features closed and open longitudinal slots (Fig. 4). The starboard-side of the model is mounted to the tunnel wall (ceiling) with the aid of peniche. The incidence angle of the test article is changed by rotating the model geometry together with peniche inside the test section.

The important simplification done to the mathematical model of ETW is that the common plenum chamber is separated into two parts. This was done to reduce the number of cells of the computational grid. 


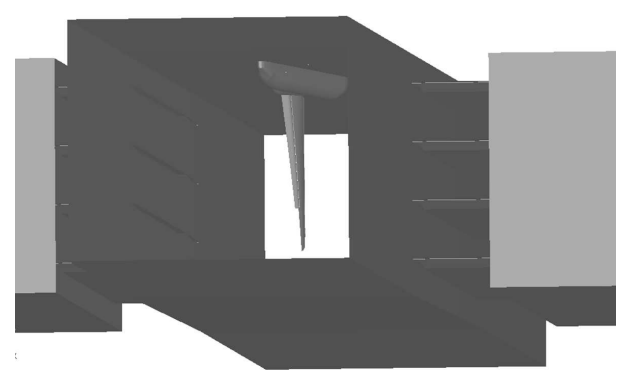

(a)

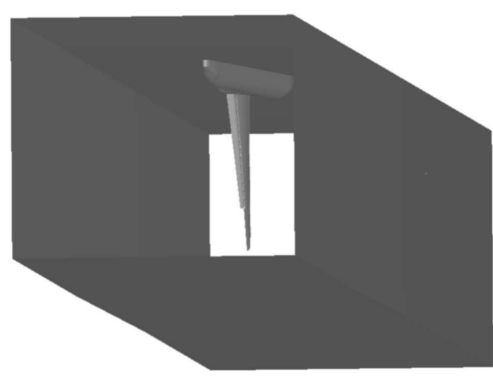

(b)

Figure 4 The ETW test section: (a) with open slots and plenum chambers; and (b) with closed slots

During the calculations, the set of parameters and reference values were used: wing area $S=0.45 \mathrm{~m}^{2}$ and mean aerodynamic chord $(\mathrm{MAC})=0.3 \mathrm{~m}$. All sizes correspond to the wind tunnel model. The origin of the coordinate axis system is depicted at the left lower corner of ETW test section. The $X$-axis is directed downstream, the $Y$-axis is located in the symmetry plane in upper direction, and the $Z$-axis is oriented from the left to the right wall. The point of model rotation has coordinates $x=3.677 \mathrm{~m}, y=2 \mathrm{~m}$, and $z=1.2 \mathrm{~m}$ and is located at the upper wall. The nose of the model at zero incidence is located at $x=2.241 \mathrm{~m}, y=1.9556 \mathrm{~m}$, and $z=1.2154 \mathrm{~m}$. The peniche thickness amounts to $0.0444 \mathrm{~m}$. The divergence of the lower wind tunnel wall equals $0.55^{\circ}$ with open slots and $0.35^{\circ}$ with closed slots.

\subsection{Grid Generation}

For the present work, a multiblock structured grid approach has been selected. The structured grid topology was adjusted in the way that allows to minimize the efforts needed for grid rebuilding due to angle of attack changing. The computational domain was divided in three independent parts:

(1) central region which includes the part of fuselage and high-lift wing;

(2) outer region, which differs for free flow and in-tunnel configurations:

(a) in case of free-flow, it contains nose and rear parts of fuselage; and

(b) in case of intunnel conditions, it contains nose and rear parts of fuselage, peniche, and wind tunnel ceiling; and

(3) ETW test section outer region.

So, in free-flow calculations, parts 1 and 2a were used for grid generation, and in in-tunnel calculations, parts $1,2 \mathrm{~b}$, and 3 were used. Thanks to this, grid in 


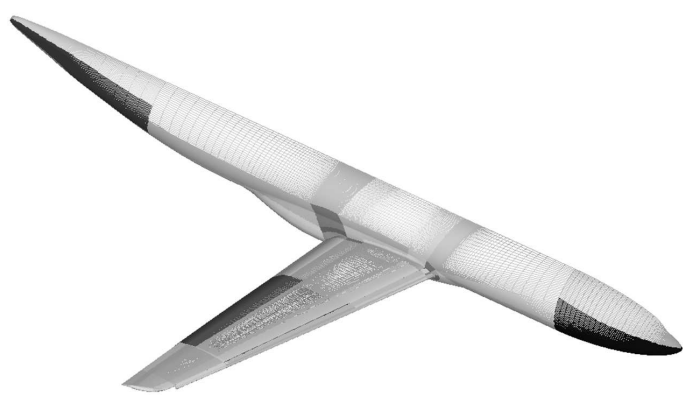

Figure 5 Surface grid for the reference configuration

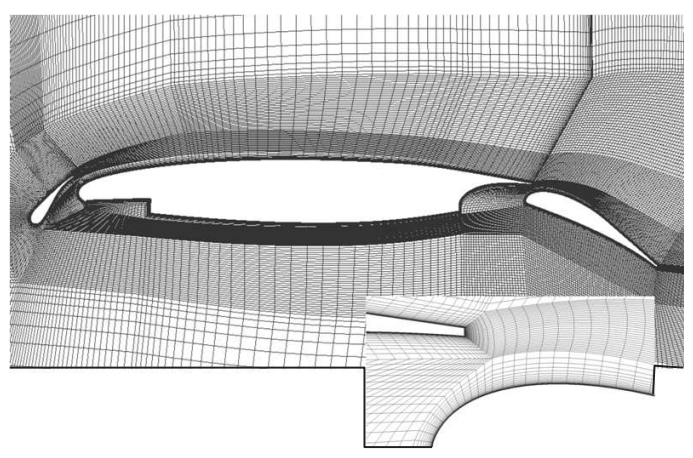

Figure 6 Example of the computational grid

parts 1 and 3 remains unchanged with the angle of attack changing. Furthermore, it allows to use the same grid topology around the wing for all configurations.

The computational grid for the model in a free air environment contains 14.5 million cells. In case the test section of the wind tunnel is included in the simulations, the grid contains 20 million cells. The decomposition of the flow domain is based on 644 blocks for the model in free flow, 688 blocks for the wind tunnel test section without slots, and 714 blocks for the wind tunnel test section with slots.

The surface grid for the high-lift configuration is shown in Fig. 5.

The number of cells along with wing span was 170, around the Krueger flap profile 120 , around the central section of wing 250 , and around the trailing edge flap 150. The boundary layer contained 33 cells.

The distance of the test article to far-field boundaries of the computational domain was not less than $4 \mathrm{~m}$ where it is noted that the wing semispan amounts to $b / 2=1.5 \mathrm{~m}$.

The calculation grid section at the middle of the wing is presented in Fig. 6 . The grid of O-type was created around all the elements. The most refinement of the grid was achieved above the wing and under the wing, especially, near gaps and wake locations.

\subsection{Test Cases}

The test cases were chosen on the base of the project requirements. The Mach number $\mathrm{M}=0.2$ corresponds to takeoff and landing regimes. Initially, the lift curve is calculated in a free flight environment. The incidence angles are 
equal to: $\alpha=0^{\circ}, 5^{\circ}, 10^{\circ}, 12^{\circ}$, and $14^{\circ}-25^{\circ}$ (step is $1^{\circ}$ ). The total pressure equals $P_{0}=333633 \mathrm{~Pa}$ and the total temperature amounts to $T_{0}=115 \mathrm{~K}$. These parameters correspond to a free-stream Reynolds number $\operatorname{Re}=16.4 \cdot 10^{6}$ (using mean chord). The static gas parameters are equal to $P=324457 \mathrm{~Pa}$, $T=114.09 \mathrm{~K}$, and $V=42.826 \mathrm{~m} / \mathrm{s}$.

The turbulence level was chosen on the basis of ETW recommendations and was equal to $T_{u}=0.7 \%$ in the vicinity of the model. Using the known scale, it was calculated that $k=1.69 \mathrm{~m}^{2} / \mathrm{s}^{2}$ and $\omega=555.55555 \mathrm{~s}^{-1}$ in the far field and $k=0.17 \mathrm{~m}^{2} / \mathrm{s}^{2}$ and $\omega=68 \mathrm{~s}^{-1}$ in the model vicinity. In the case of the wind tunnel, these parameters were prescribed as $k=0.12 \mathrm{~m}^{2} / \mathrm{s}^{2}$ and $\omega=50 \mathrm{~s}^{-1}$. Flow around the model was assumed to be fully turbulent and no transition model was used.

The flow calculations for the model installed in the wind tunnel were performed for incidence angles $\alpha=0^{\circ}, 10^{\circ}, 14^{\circ}, 18^{\circ}, 19^{\circ}, 20^{\circ}$, and $21^{\circ}$ both with open and closed slots.

It should be noticed that in both free-air and in-tunnel computations, the same flow conditions were used.

\section{RESULTS}

\subsection{Numerical Results and Experimental Data Comparison}

The convergence of the solution was controlled by the following parameters: lift coefficient, friction drag coefficient, and density residual in terms of the $L_{1}$-norm (maximal difference of density values in all cells of computational domain for two iterations). The aerodynamic force coefficients converged to a steady state at 14000 iterations from the initial uniform flow.

The aerodynamic tests of the high-lift aircraft model were performed in ETW with closed walls configuration. The wind-tunnel wall surface pressure coefficient distributions for the angle of attack $\alpha=18^{\circ}$ are displayed in Fig. 7. The agreement between numerical and experimental data is good, the deviation between data is highest at the right side wall (corresponds to lower wing surface), on which the numerical simulations predict higher pressure than measured.

A comparison between the computed and experimental uncorrected forces can be seen in Fig. 8. A very good agreement is obtained. The maximum lift is predicted at the angle of attack $\alpha=19^{\circ}$ which also coincides with the experimental location. The level of the lift is again predicted well for this angle. It should be noticed that experimental data were obtained after all computations were ended (no tuning was used).

The pressure coefficient plots at the angle of attack $\alpha=18^{\circ}$ in Fig. 9 also show good agreement between experimental and numerical pressure distributions, although the computed values of pressure at 16 percent span section, which is 

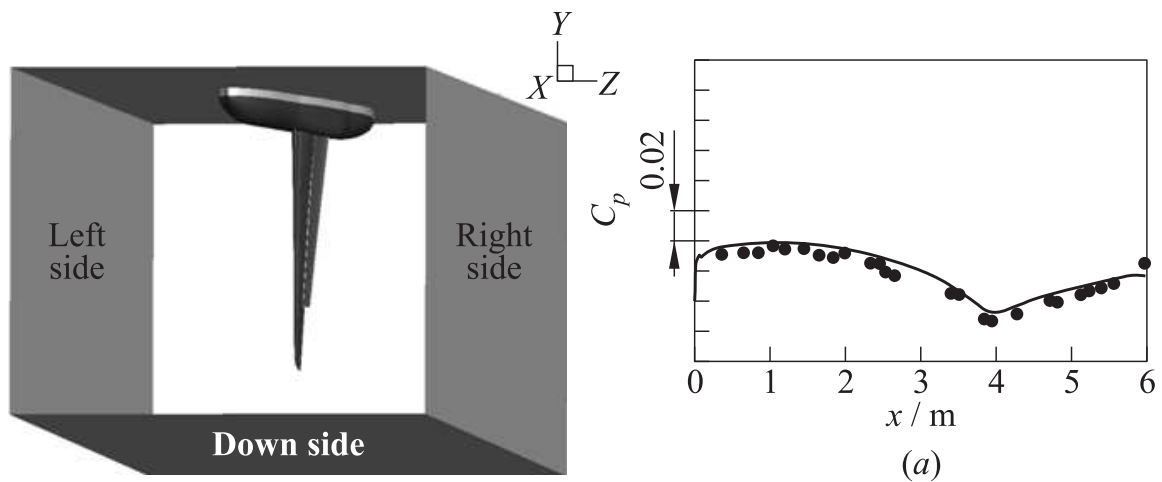

(a)

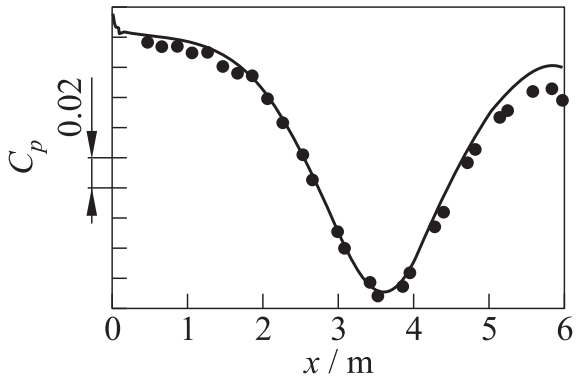

(b)

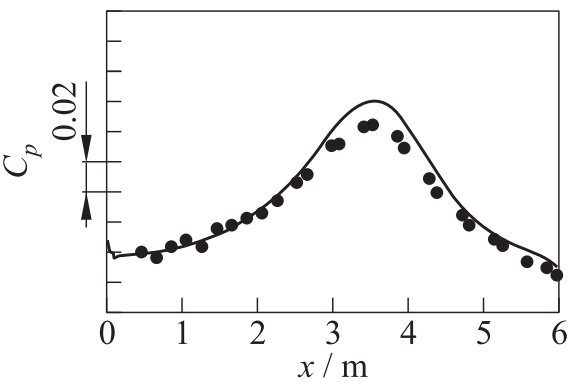

(c)

Figure 7 Pressure coefficient distribution on wind tunnel walls at $\alpha=18^{\circ}$ : $(a)$ down side wall; $(b)$ left side wall; and $(c)$ right side wall. Curves refer to computational fluid dynamics (CFD) calculations in ETW-TsAGI and signs to experiments in ETW

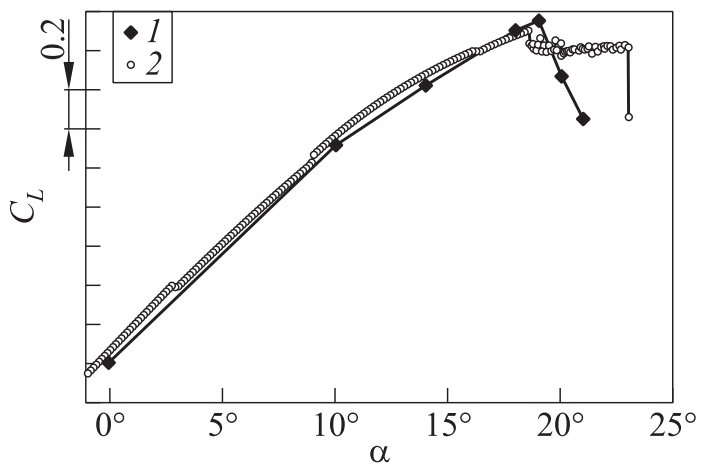

Figure 8 The comparison of experimental and calculated lift coefficients in wind tunnel with closed walls: 1 - CFD ETW-TsAGI and 2 - experiment in ETW 


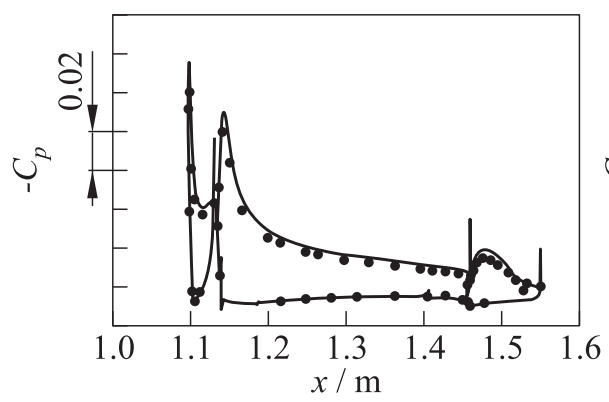

(a)

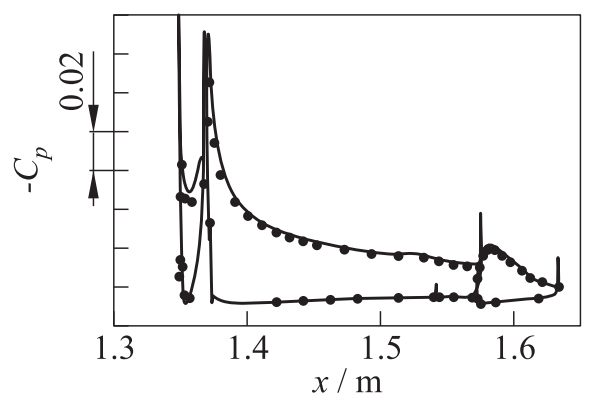

(c)

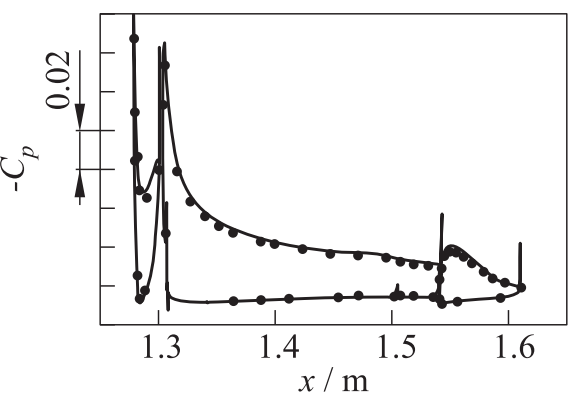

(b)

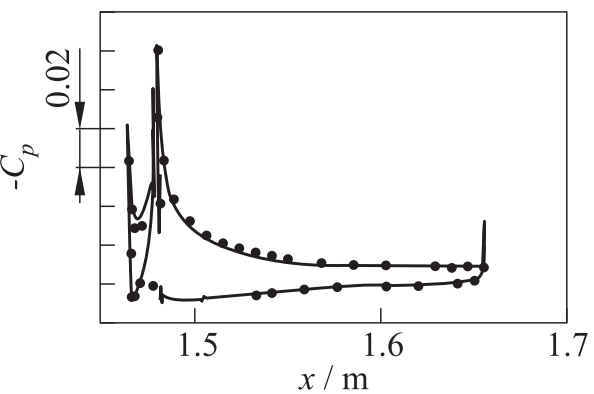

(d)

Figure 9 Comparison of pressure distributions, uncorrected experimental values (signs) and numerical results inside the tunnel, closed slots (curves): $(a) \eta=16 \%$; (b) $50 \%$; (c) $62 \%$; and $(d) \eta=83 \%$

inboard part of a wing, is slightly underpredicted. This may be caused by the fact that the thickness of the boundary layer in computations was not controlled. As a result, the computed boundary layer may differ from the real. At the tip wing section (83 percent span), the separation region could be observed both in experimental and computational data.

To summarize, the agreement between computed and measured data is very good up to the maximum lift when comparing uncorrected experiments with in-tunnel numerical results. The next step is to indicate the wind tunnel walls infuence.

\subsection{Wind Tunnel Walls Influence}

A comparison of Mach number contours at the $y=$ const plane, corresponding to the upper longitudinal wind tunnel slot for closed and slotted wind tunnel walls at $\alpha=19^{\circ}$ can be seen at Fig. 10. Figure $10 b$ which corresponds to the slotted wind tunnel walls configuration, strong cross flow from the test section 


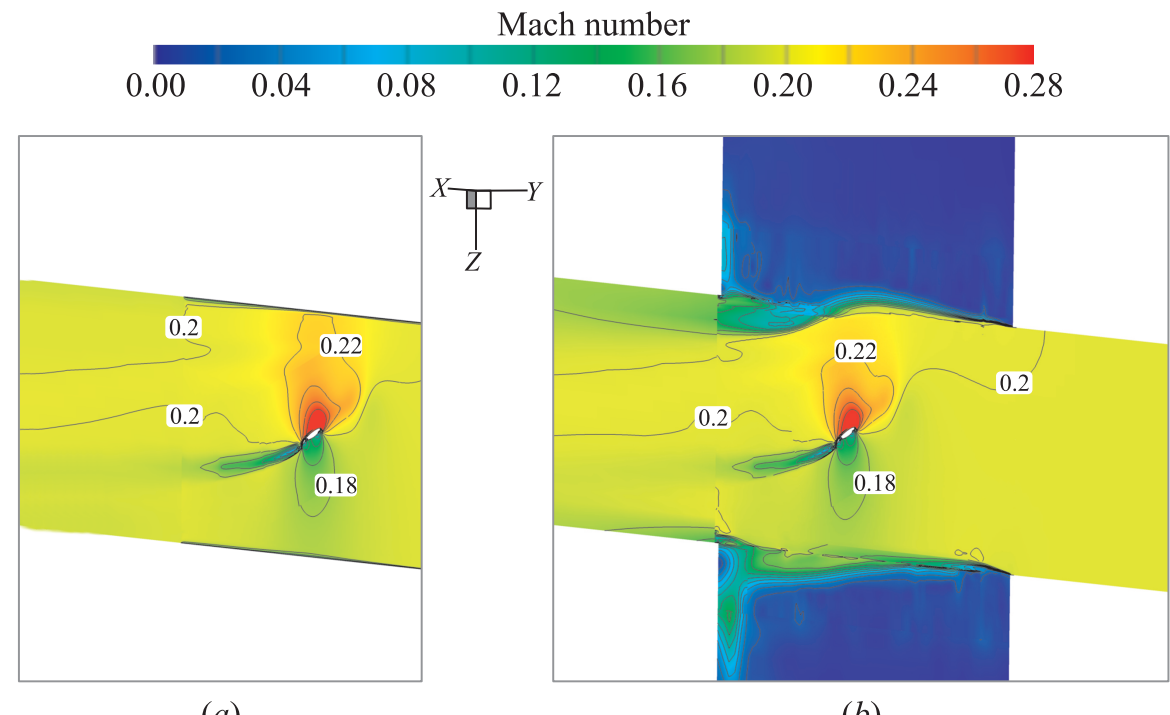

(a)

(b)

Figure 10 Mach number contours at the $y=$ const plane, corresponded to upper longitudinal wind tunnel slot for $\mathrm{M}=0.2, \operatorname{Re}=16.4 \cdot 10^{6}$, and $\alpha=19^{\circ}:(a)$ closed walls; and $(b)$ slotted walls

to the plenum chamber and vice versa can be observed. One can see that closed walls (see Fig. 10a) cause more intense acceleration of the flow above the upper side of the wing - the region bounded by the isoline $\mathrm{M}=0.22$ in Fig. $10 a$ is greater than the one in Fig. 10b. This region of disturbed flow is propagated up to the wind tunnel walls.

In the case of positive incidence angles, the presence of wind tunnel walls leads to an increase of the lift coefficient (Fig. 11) due to contraction and acceleration of flow above the wing and due to deceleration from below. On the contrary, the influence of walls results in lift diminishing when the stall takes place.

In the case of critical regime, this additional contraction of flow results in decrease of stall angle. For the given aircraft model, the stall angle diminishes for about $\Delta \alpha=1^{\circ}$ in wind tunnel with open slots and for about $\Delta \alpha=2^{\circ}$ in wind tunnel with closed slots.

In Fig. 12, the computed wind tunnel wall induced lift (i.e., the difference between the lift coefficients for in-tunnel and free-flight configurations) is shown. According to numerical results, the slotted wall configuration produces less wall interference than the closed wall configuration. This seems plausible as slotted walls are designed to reduce the wall interference.

The angle of attack growth results in the diminishing of flow area in wind tunnel test section. This causes the increase of the wind tunnel wall induced 


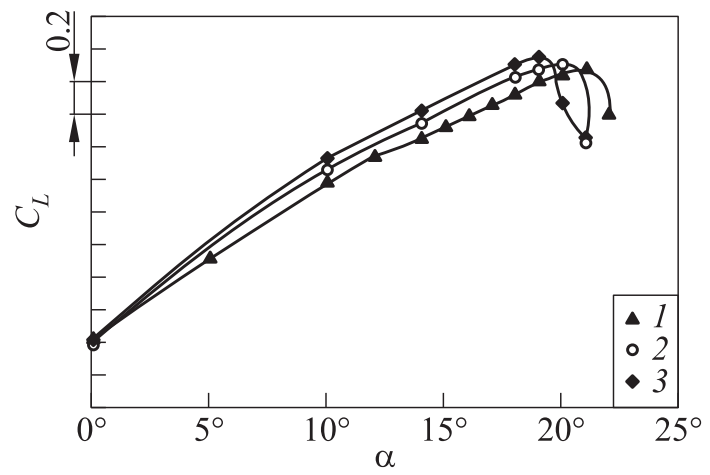

Figure 11 Lift coefficient for three configurations: 1 - free flight; 2 - wind tunnel with open slots; and 3 - wind tunnel with closed slots

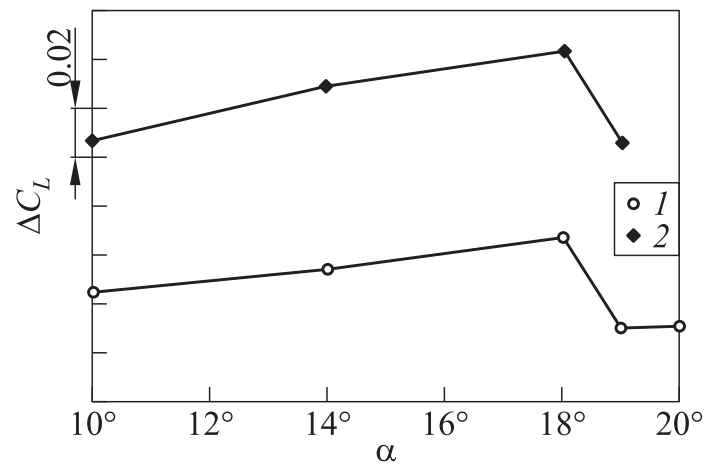

Figure 12 Wind tunnel walls induced lift: 1 - wind tunnel with open walls; and 2 - wind tunnel with closed walls

lift. As a result, the direct relation between the angle of attack and wall-induced lift is expected. Computations showed (see Fig. 12) that the wall-induced lift increments with the lift coefficient growth on the "linear" region but in the vicinity of maximum lift, the dependence is opposite.

\section{CONCLUDING REMARKS}

Flow calculations for investigation of aerodynamic performance of the DeSiReH designed high-lift laminar wing at free flight and ETW in-tunnel conditions are performed.

The agreement between computed and measured data is very good up to the maximum lift when comparing uncorrected experiments with in-tunnel numerical 
results. The numerical results show that for the given aircraft model, the stall angle diminishes for about $1^{\circ}$ in wind tunnel with open slots and for about $2^{\circ}$ in wind tunnel with closed slots compared to the free-flight conditions.

The aerodynamic coefficients of the model installed in wind tunnel with open slots is closer to aerodynamic coefficients of isolated model than those obtained for model installed in wind tunnel with closed slots.

\section{ACKNOWLEDGMENTS}

This work was partly supported by the European Commission under Contract ACP8-GA-2009-233607 and carried out within the project DeSiReH. The authors are thankful to the European Transonic Wind Tunnel (ETW) for supplying windtunnel data and geometry. The authors also would like to thank Hans Maselsnd (NLR) for comments and valuable remarks while discussing the results of current research.

\section{REFERENCES}

1. Rudnik, R. 2003. CFD assessment for 3D high lift flows in the European Project EUROLIFT. AIAA Paper No. 2003-3794.

2. Eliasson, P. 2003. CFD improvements for high lift flows in the European Project EUROLIFT. AIAA Paper No. 2003-3795.

3. Wild, J., J. Brezillon, O. Amoignon, J. Quest, F. Moens, and D. Quagliarella. 2007. Advanced high-lift design by numerical methods and wind tunnel verification within the European Project EUROLIFT II. AIAA Paper No. 2007-4300.

4. Eliasson, P. 2008. Investigation of a half-model high-lift configuration in a wind tunnel. J. Aircraft 45(1):29-37.

5. Bosnyakov, S., I. Kursakov, A. Lysenkov, S. Matyash, S. Mikhailov, V. Vlasenko, and J. Quest. 2008. Computational tools for supporting the testing of civil aircraft configurations in wind tunnels. J. Prog. Aerosp. Sci. 44:67-120.

6. Bosnyakov, S., I. Kursakov, S. Mikhaylov, V. Vlasenko. 2014. Calculations of unsteady flows around high-lift configurations based on a zonal approach. Progress on flight physics. Eds. D. Knight, Ph. Reijasse, and I. Lipatov. EUCASS advances in aerospace sciences book ser. Moscow-Paris: TORUS PRESS-EDP Sciences. 7:4566 . 